\title{
HEMODYNAMIC FEATURES ASSOCIATED WITH ABDOMINAL AORTIC ANEURYSM (AAA) GEOMETRY
}

\author{
Guillermo Vilalta (1), Eduardo Soudah (2), José A. Vilalta (3), Félix Nieto (1), Maurizio \\ Bordone (2) María Á. Pérez (4), Carlos Vaquero (5)
1. CARTIF Centro tecnológico, Valladolid-Spain, 2. International Center for Numerical Methods in Engineering (CIMNE), Barcelona-Spain, 3. Polytechnical University of Havana, Havana-Cuba, 4. Lou Institute, University of Valladolid, Valladolid- Spain, 5. University and Clinic Hospital of Valladolid, Valladolid-Spain.

\section{Introduction}

Recent findings have shown that maximum diameter of abdominal aortic aneurysm (AAA) and its growth rate are not entirely reliable indicators of rupture potential. The AAA geometrical shape and size may be related to the rupture risk which is a clinical manifestation of the balance between the forces generated by blood flow within the AAA and its strength. This study aims at assessing the hemodynamic features associated with the geometry of AAAs.

\section{Methods}

Twenty eight parametrized AAA models were generated in CATIA V.5R19 based on geometries proposed by [Scotti, 2005]. In order to optimize the number of the models, a factorial combination using the main AAA geometric parameters (maximum diameter, length and asymmetry) in 3 different levels ( $3^{3}$ factorial) has been carried out. The computational grid mesh was generated using the graphical pre and post processor for computer simulation and analysis, GiD [GiD]. Navier-Stokes equations, with the assumptions of incompressible and Newtonian fluid with a density of $1050 \mathrm{~kg} / \mathrm{m} 3$ and a kinematic viscosity of 4 10-3 Pa.s were used in this work. The boundary conditions used for whole 28 models were time-depended conditions. Two waveforms taken from previous studies have been selected to reproduce in vivo pulsatile velocity (prescribed at the abdominal aorta inlet) and pressure (prescribed at the abdominal aorta outlet). A rigid arterial wall was assumed. A framework for building multi-disciplinary finite elements program Kratos [Kratos, 2011] was used for solving the Computational fluid problem.

\section{Results and Discussion}

The flow patterns are highly disturbed with recirculating regions within the sac. This behaviour may be considered as a combination of rotational and recirculating secondary flow. During early phase of the velocity pulse prescribed $(t<0.2 \mathrm{~s})$, the flow patterns are characterized by low negative velocities at distal neck, close to the wall, due to convective effects as the flow adapts to changes of the aneurysm geometry sac. When systolic acceleration occurs $(0.2<t<0.4 \mathrm{~s})$, an increase of the recirculating region is verified occupying almost the aneurysmatic sac that cause the ejection of the vortices existing at onset of the cardiac cycle. The systolic deceleration is characterized by flow separation forming two new vortices at proximal neck. A high velocity internal jet that travels by AAA centreline is subjected to significant hemodynamic disturbance. In the period of flow reversal, the vortices core moves towards the centerline, because of the decreasing of the intensity of recirculating flow. Finally, at the beginning of the diastole, these vortices move towards midsection of sac, an intensive and axisymmetric recirculation is present close to the aneurysm distal end and also a negative velocity appears along anterior wall. The results of the time dependent flow field computation shows that most of the AAA lumen wall surface was exposed to very low wall shear stress (WSS). Locally elevated WSS were located close to the proximal and distal neck [Biasetti, 2011]. The peak values of WSS are obtained during systolic acceleration, and the wall pressure distribution is almost uniform at $t=0.37 \mathrm{~s}$. The AAA asymmetry causes that the complex flow patterns are intensified, mainly due to the effect of the changes that occur in the behavior of the internal jet of blood flow as consequence of the asymmetry of the flow field in aneurysmatic sac. For AAA shorter models, the effect of the recirculating regions in the aneurysm sac is more intense. With the increase of the length, these regions tend to remain in the aneurysm midsection until the velocity pulse reaches its peak value. Then, the vortices are ejected of the sac.

Therefore, CFD simulations have shown that exist a highly dependence on flow-induced hemodynamic stresses due to AAA geometry. And, for that reason, the AAA geometry may be established as contributing factor to the potential rupture.

\section{References}

J. Biasetti, J.R. Soc. Interface, 8: 1449-1461, 2011. C.M Scotti, Biomedical Eng. OnLine, 4:64, 2005.

GiD. The personal pre and postprocessor, http://www.gidhome.com CIMNE (2011).

Kratos: Framework for building multi-disciplinary finite element programs CIMNE (2011). 